\title{
El uso de la pizarra digital interactiva en el proceso enseñanza-aprendizaje en la Educación Superior
}

\author{
Antonia Larissa Hernández Monterrosa \\ Máster en Profesionalización de la Docencia Superior \\ Investigadora-docente \\ Dirección de investigación \\ Universidad Católica de El Salvador \\ Email: larissa.hernandez@catolica.edu.sv
}

Recepción: 20-11-2014 / Aceptación: 17-01-2015

\section{Resumen}

En lo concerniente a tecnologías de información y comunicación (TIC), existe una variedad de recursos potencialmente utilizables para hacer el desarrollo del proceso de enseñanza aprendizaje más dinámico e interesante. La pizarra digital interactiva (PDI) es una opción relativamente nueva en el contexto educativo, de la cual existen una amplia variedad en el medio: pizarras digitales táctiles con malla; pizarras digitales electromagnéticas con lápiz; pizarras digitales táctiles por infrared; y pizarras digitales por ultrasonidos-infrarroja con lápiz. Sobre esta última se estudió su funcionamiento como recurso didáctico, además de su implementación en el desarrollo de metodologías que promuevan la innovación pedagógica. También se verificaron aspectos cuantitativos y cualitativos que permitieran visualizar el rendimiento académico de los estudiantes. Para ello se empleó el estudio de caso evaluativo como metodología de investigación.

Se concluye que, la PDI es un recurso que ayuda al aprovechamiento de las clases, ya que permite la creación de actividades como mapas conceptuales, inserción de documentos, videos e imágenes para que los estudiantes interactúen y su aprendizaje sea significativo. Otro aspecto importante es que al usar este tipo de recursos, el docente mejora su práctica pedagógica.

Palabras clave: Pizarra digital interactiva, tecnología, innovación, metodología

\begin{abstract}
Concerning to information and communication technologies (ICT), there is a variety of resources that can be used to make the development of the teaching-learning process more dynamic and interesting. The interactive digital board (IDB) is one option relatively new in the educational context from which there is a wide variety in the mean: tactile-digital board with a mesh, electromagneticdigital board with pencil, tactile-digital board by infrared and digital board by ultrasound-infrared with pencil. About that last one, it was studied its functioning as a didactic resource and even its implementation in the development of methodologies that foster the pedagogic innovation. Moreover, it was verified the qualitative and quantitative aspects that let visualize the academic performance of the students. For this, the evaluative case was implemented as methodology of investigation.

It concludes that the IDB is a resource that helps to take advantage of the classes because it allows to create activities like concept maps, insertion of documents, video and images for the students to interact and make their learning meaningful. Another important aspect is that when using this type of resources, the teacher improves his pedagogic practice.
\end{abstract}

Key words: Interactive-digital board, technology, innovation, methodology 


\section{Introducción}

Actualmente, el ser humano se está inmerso en una sociedad que avanza cada vez más deprisa y en donde los conocimientos se multiplican a gran velocidad. Esta situación repercute de forma ineludible en el proceso de enseñanza aprendizaje de todos los niveles educativos, pero con mayor énfasis a nivel universitario, en donde se prepara a los futuros profesionales. El papel que las tecnologías de la información y la comunicación (TIC) cobran en las aulas es vital, ya que ayudan a que los contenidos se compartan eficientemente entre los estudiantes.

Un recurso innovador que ha cambiado el desarrollo de las clases es la Pizarra Digital Interactiva $(\mathrm{PDI})^{1}$. Esta es una herramienta didáctica que ayuda a que el aprendizaje se vuelva más participativo. Su utilización tiene muchas ventajas, tales como: facilitar una mayor oportunidad para la interacción y el debate en aula; versatilidad como recurso, acceso a aplicaciones para todas las áreas curriculares y aumento de la disponibilidad de tiempo, permitiendo al docente presentar con facilidad y eficacia recursos de internet o de otra fuente informática. El uso de esta tecnología no debe ser indiferente para los docentes, ya que ella permite mejorar las habilidades, destrezas y el rendimiento académico de los estudiantes.

$\overline{1 . \text { A partir de este }}$ punto, la autora se referirá al término mediante sus siglas.
La PDI, en el aula de clases, abre una ventana al mundo de la innovación y la cooperación, permitiendo compartir y comentar todo tipo de materiales, trabajos seleccionados o realizados por los docentes y discentes. Además de brindar la posibilidad de comunicarse e interactuar entre ellos en tiempo real (sincrónico) dentro de la misma clase y/o con personas ajenas a ella.

De esta manera, las metodologías que promueven la innovación pedagógica, con el uso de la pizarra digital en el proceso enseñanza aprendizaje, procuran hacer cambios en las formas de enseñanza de los docentes. Graells, (2006) lo expone de la siguiente manera: “induce una notable renovación de las metodologías docentes y de los procesos de enseñanza y aprendizaje; incrementa la motivación de los estudiantes, revitaliza la autoestima profesional de los profesores y facilita el logro de los aprendizajes más significativos, acordes con la sociedad actual” (pág. 12).

\section{Origen de las PDI}

Históricamente, la primera pizarra de este tipo "se fabricó por SMART Technologies Inc. en 1991" (Hernández y Marín, 2011, pág. 3); desde entonces, se ha reconocido que esta tiene gran potencial como herramienta en el proceso enseñanza aprendizaje. Actualmente, es un recurso que ha innovado el proceso, especialmente en lo que respecta a la práctica docente, el interés y la motivación del alumnado en las aulas de clase. 
Con respecto a la conceptualización de la PDI, varios autores tienen su propia perspectiva. Algunos la definen como: "una pantalla sensible de diferentes dimensiones que, conectada a un ordenador y a un proyector, se convierte en una potente herramienta en el ámbito de la enseñanza" (Ministerio de Industria, Turismo y Comercio, 2006, pág. 4). Otra definición que plantea Medina (2011) es considerar a la PDI como "la unión de tres elementos fundamentales: un ordenador multimedia con posibilidad a Internet, un video-proyector y una pizarra -que podrá ser táctil o no dependiendo del modelo-, donde se proyectarán los contenidos educativos" (pág. 1).

Existen en el medio tecnológico diferentes pizarras digitales interactivas, cuyas características varían según cada una de ellas. A continuación se detallan algunas de sus diferencias:

a) Pizarras digitales táctiles con malla. Permiten interactuar de una forma táctil con los dedos y también con un lápiz. Ejemplos: TeamBoard, Traceboard, SmartBoard y IQBoard.

\section{b) Pizarras digitales electromagnéticas} con lápiz. Estas utilizan una malla de cables de cobre dentro de ella, que cubren toda la superficie para captar la señal de un lápiz especial. Ejemplos: Promothean, Interwrite y Clasus.

\section{c) Pizarras digitales táctiles por infrared.} Estas tienen las características de todas las anteriores, pero usan una tecnología diferente, ya que muchas son magnéticas. Para ellas, se pueden utilizar rotuladores especiales para pizarras blancas. Ejemplos: Hitachi Starboard FX Duo, FXTRIO y TouchIT.

\section{d) Pizarras digitales por ultrasonidos-} Infrarroja con lápiz. Se trabaja con un dispositivo que se coloca en una pizarra blanca ya existente, ayudado por un lápiz óptico de baterías. Cuando el lápiz entra en contacto con la superficie de la pizarra, envía simultáneamente una señal ultrasónica y otra de tipo infrarrojo para establecer sincronía. Ejemplo: eBeam y Mimio (Medina, 2011, pág. 2).

Para la instalación y utilización habitual de la PDI se necesitan algunos elementos anexos, los cuales como mínimo deben incluir lo siguiente: "Ordenador multimedia, proyector, medio de conexión, pantalla interactiva, software de la pizarra interactiva"(Ministerio de industria turismo y comercio, 2006). Cabe señalar que la adquisición de una pizarra digital interactiva incluye "la pantalla, los elementos para interactuar con el ella (rotuladores, borradores, etc.); el software asociado y todo el cableado correspondiente, el proyector, la computadora, los periféricos y accesorios que se consideren necesarios" (Fernández, 2013, pág. 2). 
También se integran a las PDI otros elementos que aumentan su funcionalidad, por ejemplo: "un lector de documentos, webcam sencilla, un sistema de amplificación de sonido, con altavoces de potencia, una impresora de inyección de tinta en color, la conexión del ordenador a una antena de televisión convencional, cable o satélite” (Soto, 2011, pág. 7).

\section{Tecnologías y docencia}

Hoy en día, la actualización docente es primordial, porque cada vez más la tecnología avanza a pasos agigantados. Según lo establecido por el Real Decreto 1631/2006 en el documento aulas del siglo XXI: retos educativos Díaz, (2012), la competencia digital consiste en "disponer de habilidades para buscar, obtener, procesar y comunicar la información hasta su transmisión en distintos soportes una vez tratada, incluyendo la utilización de las tecnologías de la información y la comunicación como elemento esencial para informarse, aprender y comunicarse" (pág. 11). Por tanto, los docentes deben desarrollar esta competencia, a fin de mejorar su labor en las aulas de clase, volviéndose capaces de integrar las TIC en el momento justo.

Asimismo, cuando los profesores adquieren el conocimiento y las habilidades suficientes en el uso de la PDI, son capaces de incrementar la interactividad e implementar una variedad de recursos, debido a que ella "presenta herramientas que permiten su utilización espontáneamente, rotuladores y marcadores. Además, de la posibilidad de escribir todo tipo de texto, que puede ser rápidamente modificado; al mismo tiempo, elementos digitales tomados de la Web, fotografías, dibujos o animaciones" (Pardo, Kimelman y Villareal, 2008, pág.8). También como lo explica Gandol, Carrillo, y Prats, (s. f.), "el aprendizaje entre iguales en situaciones de necesidad por saber, es una estrategia muy efectiva; aunque generalmente son más habituales las situaciones de formación formal” (pág. 6).

Dicho de otra manera, la formación en PDI permite la adquisición de habilidades para el manejo de las TIC por parte de los docentes. No hay que dejar de lado que el buen uso de esta es capaz de cambiar la naturaleza de las prácticas pedagógicas, permitiendo adaptar los métodos de enseñanza a las necesidades individuales de los discentes.

Dentro de las ventajas de este recurso digital en el proceso enseñanza aprendizaje se encuentran:

- Mejora considerablemente los procesos de enseñanza y aprendizaje.

- Facilidad de utilización, de mantenimiento, de conservación y actualización.

- Promueve mayor participación y protagonismo del alumnado.

- Las clases resultan más atractivas y vistosas, tanto para los docentes como para los 
alumnos, ya que brinda la posibilidad de usar recursos más dinámicos y variados (sitios web, videos, audio, email, aplicaciones educativas, etc.).

- Aumenta el fomento del debate en las clases, dado que se incrementan los niveles de interacción entre el profesor, los alumnos, la materia a impartir y la tecnología utilizada.

- El uso de la pizarra optimiza el tiempo que el docente tiene para enseñar, ya que le permite usar nuevas fuentes de recursos educativos.

Tener una PDI en el aula es la posibilidad de acceder a toda clase de información digital, tal como lo explica Salinas (2009), "la pizarra digital es una puerta a internet; es la red de redes, desde la que se pueden tomar infinidad de caminos" (pág. 3-4).

Ello pone de manifiesto la versatilidad de la pizarra digital, dando lugar a una diversidad de actividades de aula, además de fomentar el trabajo en equipo y beneficiar el aprendizaje.

Gómez (2012) expone que "la PDI constituye un complemento a las metodologías y medios de enseñanza tradicionales, que se enfocan actualmente hacia un modelo de enseñanza digital e integrador" (pág. 2). Su uso en las Instituciones de Educación Superior "se impone, renueva o innova los antiguos recursos así como metodologías" (Chanto y Durán, 2012, pág. 2).
En el contexto de la Universidad Católica de El Salvador (UNICAES) ${ }^{2}$, el reproductor multimedia, la computadora y en algunos casos las tabletas son algunas de las tecnologías que se incorpora en las aulas de clase; pero es necesario ir más allá. Utilizar la PDI dentro de la institución, promueve un cambio en el proceso enseñanza aprendizaje, ya que su aplicación se encamina hacia la diversificación e incorporación de nuevas estrategias metodológicas que conduzcan al aprovechamiento de su potencial como innovación pedagógica. Tal como lo explica Hervás, Toledo, y González (2010), citando a Ekhnami (2002) y Levy (2002), "la pizarra digital interactiva es un recurso tecnológico en su totalidad pues facilita la incorporación y uso de un rango de recursos multimedia en las clases como textos, imágenes, video, sonido, diagramas, sitios webs en línea” (pág. 2).

Fue así como se formuló la siguiente pregunta de investigación ¿Qué efecto tiene el uso de la pizarra digital interactiva en el proceso enseñanza-aprendizaje en la Educación Superior?

\section{Metodología}

La investigación contempló el estudio de caso evaluativo como una estrategia para generar descubrimientos entre un grupo de estudiantes, específicamente alumnos de tercer año de la Licenciatura en Ciencias de la Educación con especialidad en Educación Parvularia

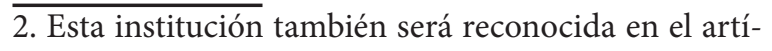
culo por sus siglas. 
de la Universidad Católica de El Salvador. La muestra se conformó de 18 estudiantes de la asignatura de Investigación Educativa.

Uno de los objetivos de la investigación fue implementar la utilización de esta pizarra en el proceso enseñanza-aprendizaje, para demostrar su funcionamiento como recurso didáctico, desarrollando metodologías que promovieran la innovación pedagógica a través de ella. Además, se verificaron aspectos cuantitativos y cualitativos que permitieron constatar el rendimiento académico de los estudiantes.

Las técnicas e instrumentos de recolección de datos que se utilizaron fueron un cuestionario y la bitácora. El primero se utilizó a manera de diagnóstico (al inicio y fin del ciclo), cuyo cuestionario final sirvió como valoración de la aplicación de la PDI. Por su parte, la bitácora sirvió para registrar la información de la observación directa participante de los alumnos.

El procedimiento de investigación se llevó a cabo en durante diversas etapas: como primera fase se planificó el estudio; luego se seleccionó el tema a investigar. Posteriormente, se buscó información relacionada a las principales variables, y se validó el protocolo de investigación junto con los instrumentos correspondientes. La segunda fase consistió en el registro de las actitudes y actividades que se realizaron durante el ciclo académico con los estudiantes de la muestra.
Respecto al procesamiento, tabulación e interpretación de la información, se utilizó el software Microsoft Office, específicamente los programas Word y Excel, para vaciar la información en matrices diseñadas para cada variable del estudio. En lo que se refiere a la interpretación de la información, ella fue sometida a un análisis inductivo que permitió comentar los datos en función de las variables que se investigaron.

\section{Resultados}

\subsection{Funcionamiento de la Pizarra Digital}

\section{Interactiva}

Los estudiantes conocían conceptualmente la pizarra digital interactiva, pero no habían tenido la oportunidad de manipularla. Cuando la PDI se instaló la primera vez en el salón de clase, ellos preguntaron ¿Qué es ese aparato?, ¿Cómo funciona?, ¿Para qué se utiliza?, mostrándose interesados en su funcionamiento.

Al utilizar la PDI en clase se notó motivación e interés de parte de los estudiantes. Puede decirse que el conocimiento adquirido fue significativo, ya que existió interacción, creatividad y participación entre ellos. Asimismo, la PDI resultó ser un apoyo didáctico, tanto para el docente como para los estudiantes, a quienes mediante ella se les facilitó tomar ideas principales de los contenidos. 


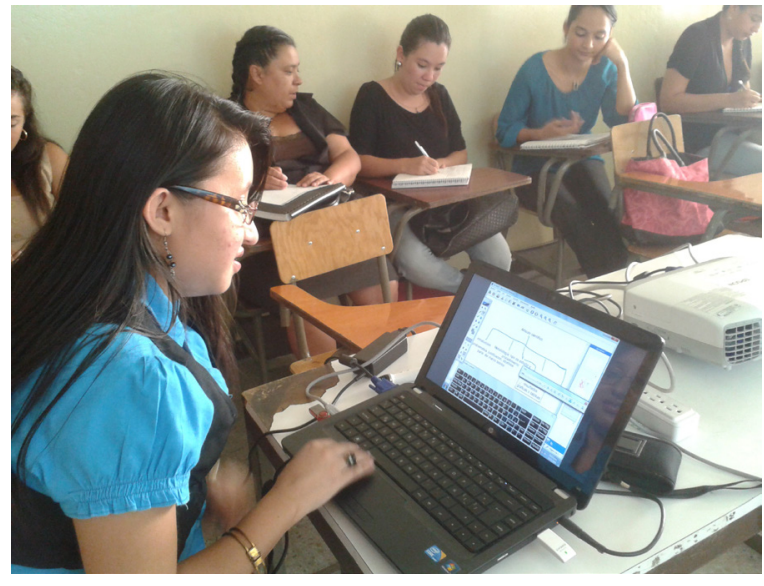

Figura1. Alumnos tomando apuntes. Funcionamien-

to de la Pizarra Digital Interactiva (Octubre, 2014).

De acuerdo a la opinión de los estudiantes, este recurso fue un excelente instrumento didáctico, ya que al ser un insumo nuevo dentro del aula, mantuvo su interés en el desarrollo de la clase, haciéndola más amena y participativa; asegurando haber adquirido más conocimientos. Algunos de ellos señalaron que, les pareció una herramienta muy llamativa de fácil manejo. No obstante, también se señalaron algunas desventajas. Algunas

de ellas enfocadas a aspectos técnicos tales como el mal funcionamiento del lápiz electrónico al momento de seleccionar un documento o marcar ideas clave; o una pobre proyección del equipo multimedia que la hiciera poco visible o inentendible (proyecciones en color amarillo, rosado, verde o morado).

Para los estudiantes, este tipo de herramientas pueden hacer que los docentes dejen de utilizar materiales concretos (folletos), para basarse solamente en TIC. Esto aunado al hecho que el estudiante o el docente no conozcan está herramienta y lo utilice mal. Otro aspecto a considerar fue el que los docentes no utilicen este tipo de recursos digitales para la clase, siguiendo con las clases tradicionales en pizarra para yeso o acrílica; o solo con diapositivas saturadas de información.

\subsection{Metodologías para la innovación pedagógica}

Con respecto las metodologías utilizadas en la clase, se involucró a los estudiantes para que interactuaran con el recurso digital, a fin que el desarrollo del proceso enseñanza aprendizaje se volviera significativo. Como resultado, se pudieron realizar diversas actividades desde la calibración de la PDI: Mapas conceptuales, creación de textos, inserción de documentos de Microsoft Power Point, Word, Excel o Acrobat PDF. También se pudo subrayar ideas principales de un texto específico, resolver sopas de letras e insertar videos e imágenes relacionados con la asignatura de Investigación Educativa.

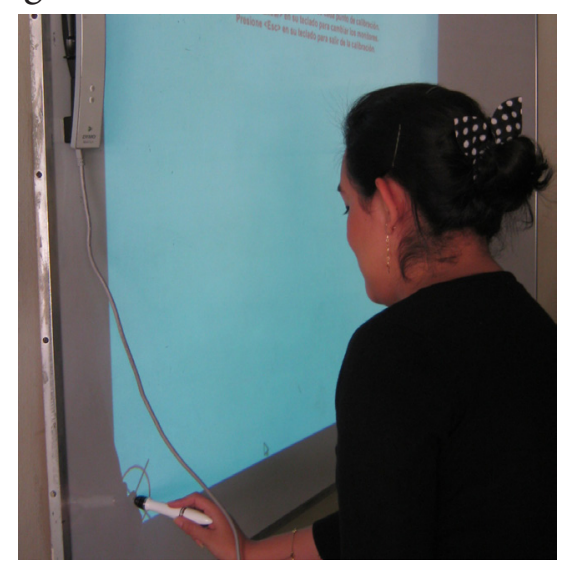

Figura 2. Calibración de la PDI. Metodologías para la innovación pedagógica (Octubre, 2014). 


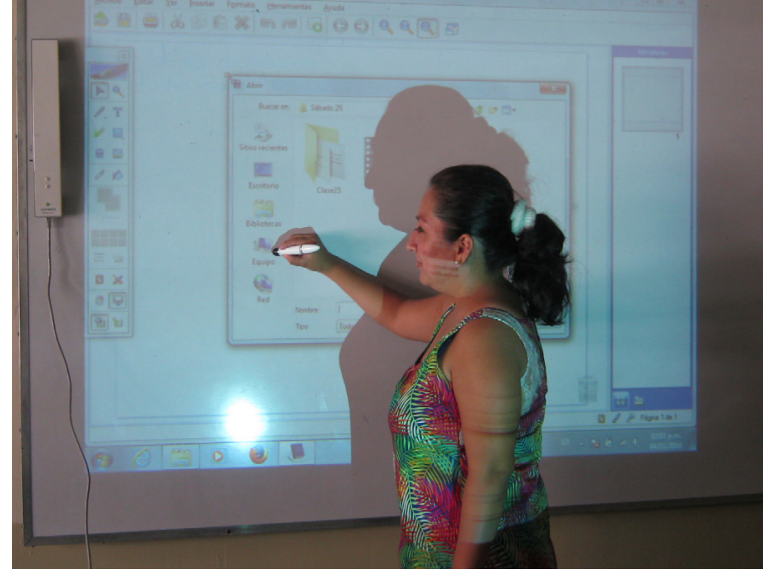

Figura 3. Inserción de documentos a la PDI. Metodologías para la innovación pedagógica (Octubre, 2014).

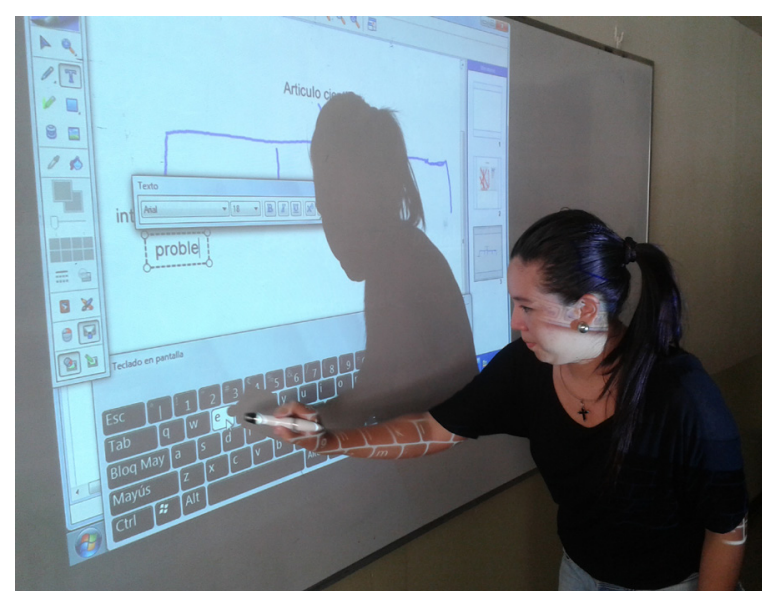

Figura 4. Elaboración de mapa conceptual. Metodologías para la innovación pedagógica (Octubre, 2014).

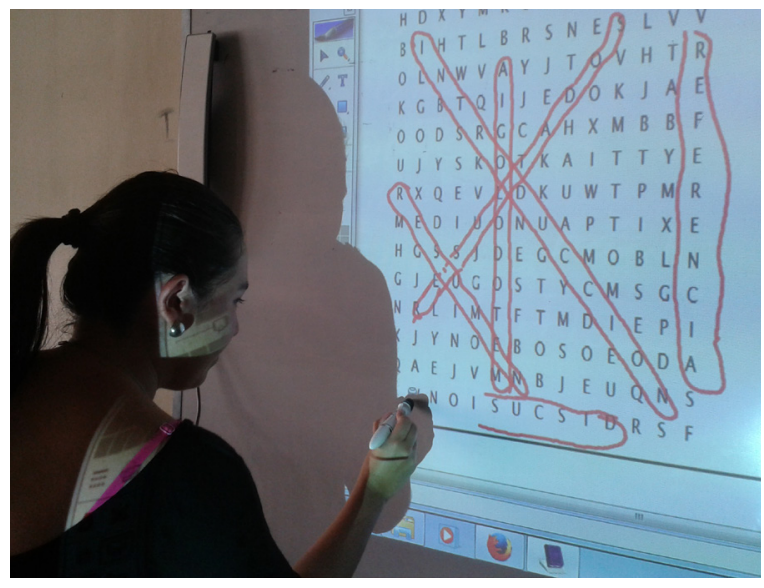

Figura 5. Sopa de Letras. Metodologías para la innovación pedagógica (Octubre, 2014).

\subsection{Actitudes ante la implementación de la PDI}

Inicialmente, la actitud observada en los estudiantes fue de curiosidad, ante el nuevo recurso (PDI) que se les presentó para desarrollar la clase. A la vez, se notó extrañez, temor e inseguridad cuando se les preguntó si alguien quería realizar alguna actividad en ella,

pues jamás habían tenido la oportunidad de verla ni mucho menos manipularla, por lo que no podían utilizarla. Cuando se les explicó el propósito de la PDI, mostraron interés y un cambio de actitud. Se observaron positivos y prestos a aprender algo nuevo con respecto a la tecnología.

Asimismo se percibió una mayor atención de parte de los estudiantes en el desarrollo de la clase. Esto fue perceptible durante la valoración final, en donde un estudiante escribió: "Las clases no fueron tediosas; al contrario, fueron más divertidas e interactivas".

Con respecto al aspecto cuantitativo, a partir del segundo periodo se verificó el cambio en el rendimiento académico de 18 estudiantes, al implementar el uso de la PDI. 


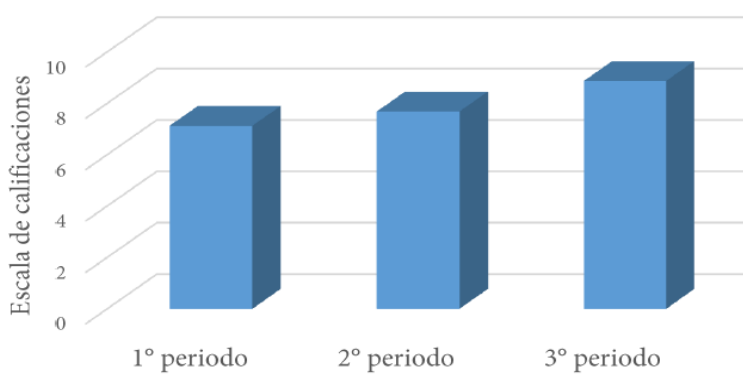

Figura 6. Promedio global de estudiantes por periodo.

Como se ve reflejado en el gráfico, el uso de la PDI fue positivo en el desarrollo de las clases por ser un instrumento novedoso, interesante y práctico para los estudiantes. Tanto así que en el primer periodo, la nota global de los estudiantes fue de 7.12; en el segundo periodo fue de $7.68 \mathrm{y}$, en el último, de 8.87. La mejora en sus calificaciones fue notable.

\section{Discusión}

La Universidad Católica de El Salvador cuenta con una PDI, cuya utilización debe aprovecharse para producir un cambio significativo en el desarrollo de las clases, así como también reconocer y visualizar a los principales beneficiarios con esta experiencia: los estudiantes.

Las Pizarras Digitales Interactivas son un recurso de potencial eficaz, eficiente y adecuado para el proceso de enseñanza aprendizaje. Es necesario impulsar este tipo de tecnología para motivar y preparar a los docentes, a fin de generar cambios en el aula, mejorar sus prácticas y el rendimiento académico de los alumnos.

Con el uso de la pizarra digital interactiva en el desarrollo de las clases de los alumnos de tercer año de la licenciatura en Ciencias de la Educación con especialidad en Educación Parvularia se obtuvo una participación más activa por parte de ello. Mediante varias actividades, se trabajó sobre la información en tiempo real, involucrando a los educandos en la actividad de compartir tareas. De esta manera se enriquecieron las actividades de tipo cooperativo, se favoreció el aprendizaje significativo; y se desarrollaron capacidades y destrezas, tanto individuales como colectivas. Un estudio realizado por la Comisión Económica para América Latina y el Caribe (CEPAL) y Claro (2010), encontró que "los estudiantes que hacen uso de las TIC, desarrollan habilidades de pensamiento de orden superior, tales como el pensamiento crítico, la resolución de problemas y la capacidad de análisis" (pág. 13). Esto debido que buscan en la Web sitios confiables para extraer información y así realizar los trabajos; al mismo tiempo, se fomenta el trabajo colaborativo entre ellos. Otro rasgo que se debe señalar es la potencialidad didáctica pedagógica de la PDI, ya que ayuda a que el alumnado presente "más motivación, y las clases sean más dinámicas y cercanas a él; facilita la corrección de ejercicios y la diversidad, el profesor puede dejar parte del material trabajado en clase en la red, es propicia para mostrar información y realizar trabajos colectivos, fomentando cooperación"(Salinas, 2009, pág. 6). 
Un elemento que es significativo en los procesos de incorporación de TIC en la enseñanza es la actitud. Mucho del éxito, aceptación o rechazo que tengan los estudiantes para trabajar con este tipo de recursos depende de ella. Tradicionalmente, como lo explica Álvarez y otros (2011), la actitud es "una disposición a reaccionar favorable o desfavorablemente hacia un objeto, situación o suceso. Las actitudes, conjuntamente con la personalidad, motivación, las expectativas de cada persona, la experiencia sociocultural o de la ansiedad, se engloban dentro de las denominadas variables afectivas de aprendizaje” (pág. 3). Tener una actitud positiva ante la incorporación de este tipo de insumos, ayuda a dinamizar el proceso enseñanza aprendizaje, ya que muchos de los docentes y alumnos no conocen sobre la existencia y la utilización del mismo. Tal como lo afirma Gutiérrez y Sánchez (s.f.), "los alumnos se motivan más ante los proceso de enseñanza aprendizaje, ya que directamente interactuar y manipular un medio, para ellos es atractivo" (pág. 10).

\section{Impacto positivo}

Los conocimientos didácticos tecnológicos que necesitan profesores y alumnos, usuarios de la pizarra digital interactiva, son importantes. Pero principalmente los profesores, son los responsables de su manejo e incorporación, para así lograr que el estudiante ponga mayor atención en el desarrollo de las clases. El docente, como lo explica Gallego, Gámiz, y Gutiérrez, (2010), "ha de planificar, impartir, tutorizar y evaluar acciones formativas, elaborando y utilizando medios y recursos didácticos, promoviendo la calidad de la formación y la actualización didáctica” (pág. 3).

De acuerdo a estos autores, algunas de las competencias que los docentes deben desarrollar son las siguientes:

- Competencias tecnológicas: saber-hacer/ utilizar las herramientas de las TIC.

- Competencias de comunicación y animación: aptitudes y capacidades Feed-back.

- Competencias psicopedagógicas: métodos de enseñanza con la ayuda de herramientas multimedia informatizadas.

En consecuencia, el profesor debe tener características que se adapten a la sociedad del conocimiento y al uso de la tecnología, como por ejemplo: "facilitador del aprendizaje, gestionar el conocimiento, dispuesto a formación permanente, usuario de recursos tecnológicos, conocedor de nuevos códigos; ágil y flexible a los cambios, creativo e innovador"(Dilac, s. f.).

Por su parte, los estudiantes deben aprender a utilizar eficaz y eficientemente el internet, en cuanto a la búsqueda de información sobre los temas de estudio; además de leer y comprender, valorar y seleccionar esta información. Como lo explica Marqués (2006), “el estudiante debe planificar, interpretar, deci- 
dir, y evaluar la información que obtiene de internet y de su entorno; y el ordenador o pizarra digital presentar, almacenar, clasificar y reproducir las actividades más rutinaria o de memoria que se le encarguen” (pág. 27). Asimismo, "cuando se trabaja con la PDI, los estudiantes reflexionan sobre la información, la relacionan con sus experiencias y sus conocimientos previos. Las posibilidades de aprendizaje activo depende en definitiva de la creatividad” (González, 2011, pág.21). En resumen, "los alumnos aumentan su motivación y satisfacción escolar; mejoran su rendimiento académico, dado que la mayoría de los alumnos consiguen alcanzar los objetivos" (García y Tejedor, 2009, pág. 12).

En cuanto a los aspectos cuantitativos y cualitativos con el uso de la PDI, estos permiten visualizar una mejora en el rendimiento académico de los estudiantes. Este elemento es definido por Alcaide (2009) como "una medida de las capacidades respondientes o indicativas que manifiesta, en forma estimativa, lo que una persona ha aprendido como consecuencia de un proceso de instrucción o formación" (pág. 5). De esta forma, se mide a través de las calificaciones que tanto los estudiantes han asimilado los contenidos desarrollados en las clases.

El uso de las tecnologías contribuye a mejorar los procesos de aprendizaje a nivel superior, porque las clases se vuelven más interactivas. Que un docente utilice este tipo de recursos como apoyo para su clase, mejora su práctica pedagógica, volviéndola una práctica innovadora, de metodologías activas que integran las Tecnologías de Información y Comunicación (TIC).

Si bien es cierto, desarrollar e implementar esta actividad supone dedicación, esfuerzo y tiempo de parte del docente, vale la pena. Los resultados que se obtienen con los estudiantes aportan una gran motivación, novedad e interactividad a la clase. En este sentido, se debe reflexionar sobre su utilización en la práctica; pero en especial sobre el desarrollo profesional que ella posibilita, brindando una metodología activa en pro del mejoramiento del proceso de enseñanza aprendizaje. 


\section{Referencias}

Álvarez, S. y otros. (2011). Actitudes de los profesores ante la integración de las TIC en la práctica docente. Estudio de un grupo de la Universidad de Valladolid. Edutec-e. Revista Electrónica de Tecnología Educativa. No.35. Valladolid: España. Recuperado de http://edutec.rediris.es/Revel2/ Revelec35/pdf/EdutecAlvarez_Cuellar_Adrada_Anguiano_Bueno_Comas_Gomez.pdf

Alcaide, R. M. (2009). Influencia del rendimiento y autoconcepto en hombres y mujeres. Revista de investigación y Docencia. Pp 27-44. Recuperado de http://www.ujaen.es/revista/reid/ revista/n2/REID2art2.pdf

Chanto, L.C y Durán, M. (2012). La pizarra interactiva como recurso en el aula. Universidad Nacional de Costa Rica. Festival Internacional de Matemática. Recuperado de http://www.cientec.or.cr/matematica/2012/ponenciasVIII/Carlos-Luis-Chanto.pdf

Claro, M. (2010). Impacto de las TIC en los aprendizajes de los estudiantes. Estado del Arte. En Sunkel, G y Trucco, D. (Coord.) de la División Social de la Comisión Económica para América Latina y El Caribe (CEPAL). Santiago: Chile. Recuperado de http://www.eclac.org/publicaciones/xml/7/40947/dp-impacto-tics-aprendizaje.pdf

Díaz, G, M. (2012). Aulas del siglo XXI: retos educativos. INTEF. Recuperado a partir de http:// www.edu.xunta.es/web/sites/web/files/aulas_del_s_xxi._nuevos_retos_educativos.pdf

Dilac, J. (s. f.). La pizarra digital: Multimedia, Interactividad y Creatividad en el Aula. Recuperado de http://lantec.fae.unicamp.br/lantec/pt/tvdi_portugues/dulac.pdf

Fernández, A. R. R. (2013). La pizarra digital interactiva como una de las tecnologías emergentes en la enseñanza actual. No 5, 15. Recuperado de http://www.3ciencias.com/wp-content/ uploads/2013/06/pizarradigital.pdf

Gallego, M. J., Gámiz, V., y Gutiérrez, E. (2010). El futuro docente ante las competencias en el uso de las tecnologías de la información y comunicación para enseñar. 34, 18. Recuperado de http://edutec.rediris.es/Revelec2/Revelec34/pdf/Edutec-e_n34_Gallego_Gamiz_Gutierrez.pdf

Gandol, F., Carrillo, E., y Prats, M. (s. f.). Potencialidades y limitaciones de la pizarra digital interactiva. Una revisión crítica de la literatura., 16. Recuperado de http://acdc.sav.us.es/pixelbit/ images/stories/p40/13.pdf 
García, A. y Tejedor, F. (2006). Evaluación de procesos de innovación escolar basados en el uso de las TIC desarrollados en la Comunidad de Castilla y León. Revista de Educación, pp 125 (147). Salamanca: España. Recuperado de http://www.revistaeducacion.educacion.es/re352/ re352_06.pdf

González, R. R, D. (2011). Impacto del uso de la pizarra digital interactiva en la enseñanza de la lectura en el grado primero en el Instituto Pedagógico Arturo Ramírez Montufar. Tesis de maestría. Universidad Nacional de Colombia. Bogotá, DC. Colombia. Recuperado de http:// www.bdigital.unal.edu.co/4375/1/04-868096.2011.pdf

Gómez, C y Márquez, F. (2012). Experiencia universitaria en el uso de la pizarra interactiva por estudiantes del título de maestro en educación especial. Congreso Virtual Internacional sobre Innovación Pedagógica y Praxis Educativa. Recuperado de http://acdc.sav.us.es/pixelbit/ images/stories/p40/13.pdf

Graells, P. (2006). Pizarra digital. Edebé. Barcelona: España. Recuperado de http://lapizarradigital.es/wp-content/manual-pizarra-digital.pdf

Gutiérrez, I. y Sánchez, M. (s.f.). Pizarra Interactiva: Usos y aplicaciones en la enseñanza. Recuperado de http://digitum.um.es/xmlui/bitstream/10201/3303/1/76.pdf

Hernández, O. y Marín, E. (2011). Pizarras digitales interactivas: una nueva forma de enseñar. Salamanca: España. Recuperado de http:// http://anpesalamanca.net/formacion/descargas/libro/Pizarra_Digital_Libro.pdf

Hervás, C., Toledo, P., y González, C. (2010). La utilización conjunta de la pizarra digital interactiva y el sistema de participación senteo: una experiencia universitaria. 36. Recuperado a partir de http://scholar.google.es/scholar?as_ylo=2010\&q=innovaci\%C3\%B3n+metodologica + con+la+pizarra+digital+interactiva\&hl=es\&as_sdt=1,5\&as_vis $=1$

Marqués, P. (2006). La pizarra digital en el aula de clase. España: edebé. Recuperado a partir de http://lapizarradigital.es/wp-content/manual-pizarra-digital.pdf

Ministerio de industria turismo y comercio. (2006). La pizarra interactiva como recurso en el aula. (p. 29). Recuperado a partir de http://www.ascmferrol.com/files/pdi_red.es.pdf 
Pardo, R; Kimelman, E y Villareal, G. (2008). Pizarras interactivas en quinto básico de inglés, proyecto de intervención y estudio. Universidad de Chile: Centro Comenius. Recuperado de http://www.enlaces.cl/tp_enlaces/portales/tpe76eb4809f44/uploadImg/File/destacados\%20cedoc/inglespizarra.pdf

Salinas, M. (2009). Análisis de la pizarra digital como recurso educativo., 4, 9. Recuperado de http://edutec.rediris.es/Revel2/Revelec45/analisis_pizarradil_recursoeducat.html

Soto, R. (2011). Características de la implantación de la pizarra digital interactiva en colegios de la comunidad de Madrid. Universidad Complutense. Madrid: España. Recuperado de http:// eprints.ucm.es/16208/1/Caracter\%C3\%ADsticas_de_la_implantaci\%C3\%B3n_de_la_pizarra_ digital_interactiva_en_colegios_de_la_Comunidad_de_Madrid.pdf 